\title{
Designing a system of photovoltaic panels for a refrigeracion center
}

\author{
Maida Bárbara Reyes Rodríguez ${ }^{1}$, Jorge Laureano Moya Rodríguez ${ }^{1}$, Jandecy Cabral Leite ${ }^{1}$ \\ ${ }^{1}$ Instituto de Tecnologia E Educação Galileo Da Amazônia - ITEGAM. Av. Joaquim Nabuco No 1950. Centro, Manaus - AM, CEP:69005-080 - Tel: \\ +559235846145. \\ E-mail: maidabrr@gmail.com, jorgemoyar@gmail.com, jandecy.cabral@itegam.org.br
}

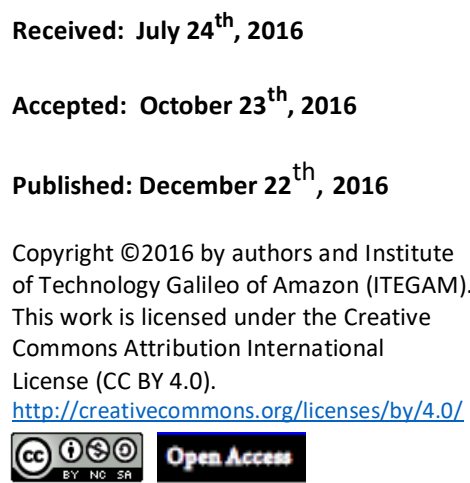

\begin{abstract}
At this paper is carried out the design of a photovoltaic system for a refrigeracion center. Taking into account the analysis of the consumption of energy of this installation in the year 2015 and at the first semester of the 2016 is defined the capacity of 2000MW-h / year to be instaled. To develop the work it was used the PVsyst V6.42 software, which allows to define the size of the installation keeping in mind the solar radiation that it receives in function of their location. The design of a friendly system of panels with the environment is achieved, since it is avoided the emission to the atmosphere of 38451 tons of $\mathrm{CO}^{2}$ in a period of 25 years and there are avoided to use 13800 tons of petroleum. Finally is carried out an economic evaluation that demonstrates the feasibility of the proposal.
\end{abstract}

Keywords: renewable energy, solr photovoltaic panels, refrigeracion center.

\section{Diseño de un sistema de paneles fotovoltaicos para um Frigorífico Diagnóstico}

\section{RESUMEN}

En este trabajo se realiza el diseño de un sistema de paneles fotovoltaicos para un frigorífico. Partiendo del análisis del consumo de energía de esta instalación en el año 2015 y el primer semestre del 2016 se define la capacidad a instalar de 2000MW-h/año. Para desarrollar el trabajo, fue usado el software PVsyst V6.42, el cual permite dimensionar el tamaño de la instalación teniendo en cuenta la radiación solar que recibe en función de su ubicación. Se logra el diseño de un sistema de paneles amigable con el medioambiente pues se evita la emisión a la atmosfera de 38451 toneladas de $\mathrm{CO}^{2}$ en un período de 25 años y se dejan de utilizar 13800 toneladas de petróleo. Finalmente se realiza una evaluación económica que demuestra la factibilidad de la propuesta.

Palavras chaves: Sinalização, Trilha, Educação Ambiental.

\section{INTRODUCCIÓN}

Toda la energía que se utilizará en el futuro vendrá del Sol; directamente a través de los módulos fotovoltaicos y colectores solares térmicos, o indirectamente en forma de viento y la biomasa. En este futuro sistema energético de la conversión y la utilización de energía será muy eficiente. Estos dos componentes, fuentes de energía renovable y eficiencia energética, son los componentes claves de la energía sostenible. La transición hacia un sistema energético sostenible es un importante reto social necesario para preservar la Tierra para las generaciones futuras [1].

En la figura 1 se muestra la capacidad anual instalada de módulos fotovoltaicos en los últimos años.Vemos que el número de sistemas fotovoltaicos instalados entre 2000 y 2011 ha crecido casi exponencialmente, con un crecimiento medio del 60\%. El crecimiento más fuerte fue entre 2007 y 2008 con un crecimiento del $143 \%$. En estos años, con mucho, la mayoría de los sistemas fotovoltaicos fueron instalado en Europa. Sin embargo, desde 2011 el número de sistemas instalados en Europa ha estado bajando rápidamente, mientras que aumenta con fuerza en las otras regiones del mundo.

Mientras que en 2011 el 74\% de todos los sistemas fotovoltaicos se han instalado en Europa, en el año 2013, esto era sólo $29 \%$. Será interesante ver cómo este desarrollo continúa en los próximos años [1]. 


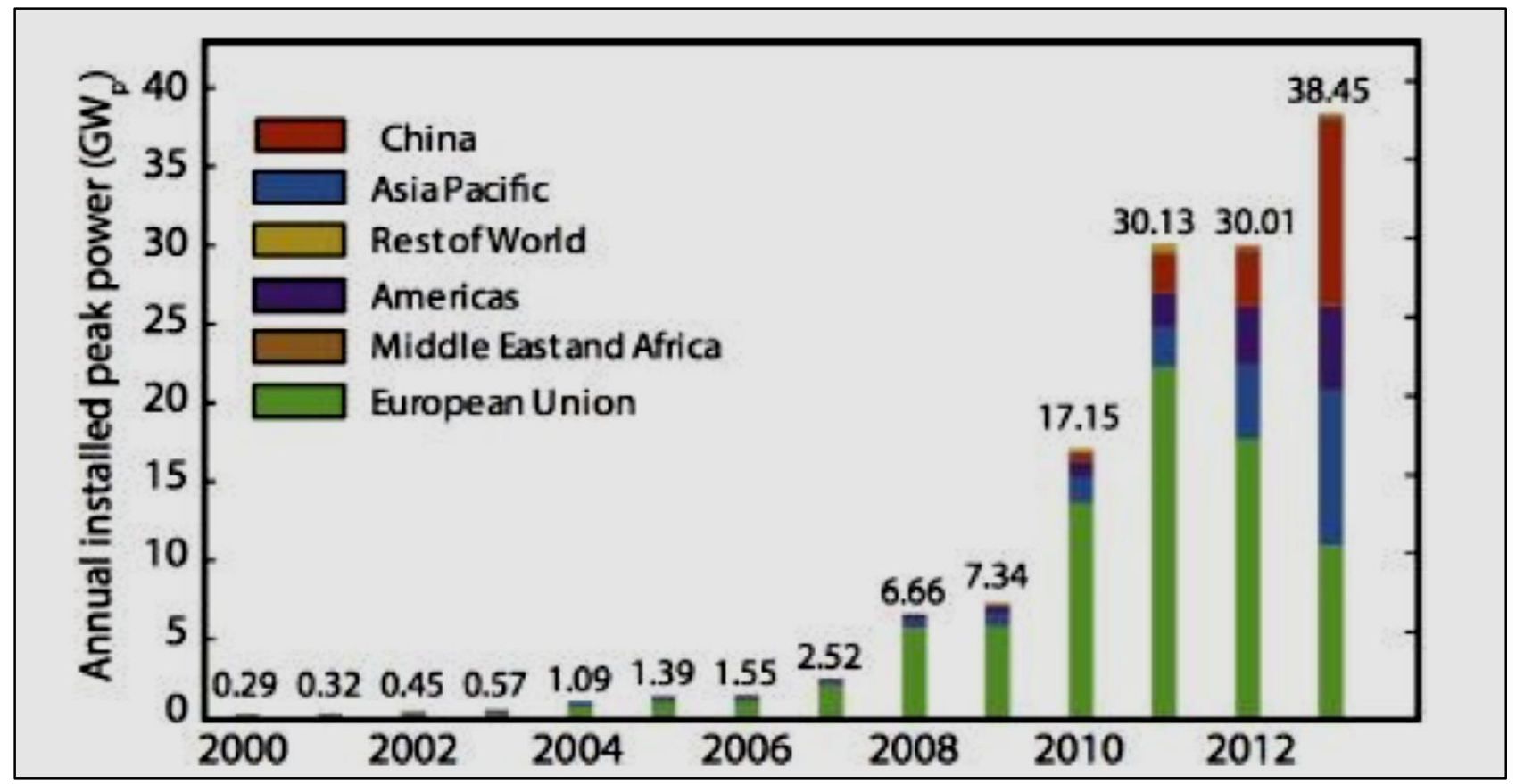

Figura 1: Capacidad Fotovoltáica instalada en años recientes.

Fuente: Isabella, Jäger et al. 2016

El aumento del consumo de energía, además de la necesidad para reducir la emisión de contaminantes en la atmósfera por el uso de fuentes de energía renovables, ha llevado a un fuerte aumento en el uso de sistemas fotovoltaicos (PV) [2].

Los sistemas solares fotovoltaicos representan un silencio seguro, fuente no contaminada, y renovable de energía eléctrica apenas consignada para la integración en el área urbana. la reducción casi completamente las pérdidas de transmisión de energía, debido a la proximidad entre la generación y el consumo [3].

La temperatura de la célula módulo PV es una función de las variables físicas del material de la celda PV, el módulo y el medio ambiente circundante. Un modelo de simulación de diferencias finitas basa en una analogía eléctrica y la descripción de un módulo fotovoltaico multi-cristalina de doble cristal ha sido desarrollado y validado utilizando datos experimentales de tal módulo fotovoltaico [4].

La energía solar puede ser reconocida como una de las fuentes de energía renovables más prometedoras, especialmente en las regiones subtropicales. Junto con otras formas de fuentes de energía renovables (es decir, el viento, geotérmica, de las olas y biomasa), que tiene un gran potencial para una amplia variedad de aplicaciones, ya de su abundancia y accesibilidad. La generación de energía fotovoltaica ha estado recibiendo considerable atención como uno de los más energías alternativas prometedoras [5].

Con el desarrollo de sistemas de generación distribuida, la electricidad renovable a partir de fuentes fotovoltaicas se convirtió en un recurso de energía en gran demanda [6].

En esos sistemas, el rendimiento de un sistema PV se basa en las condiciones de funcionamiento. Entonces, la potencia máxima extraída desde el generador PV depende en gran medida de tres factores: la insolación, perfil de carga (impedancia de carga) y la temperatura de la celda (temperatura ambiente), suponiendo una eficiencia de la celda fija [7].
El rendimiento de un panel fotovoltaico (PV) se ve afectada por su orientación y su inclinación ángulo con el plano horizontal. Esto se debe a que estos dos parámetros cambian la cantidad de la energía solar recibida por la superficie del panel PV [8].

El funcionamiento de la celda solar se basa en la capacidad de los semiconductores para convertir la luz del sol en electricidad mediante el aprovechamiento del efecto fotovoltaico. Hay un creciente interés en el estudio de los flujos de calor y masa en espacios de aire detrás de los paneles fotovoltaicos [9].

La reflexión de la radiación del sol típicamente reduce el rendimiento eléctrico de los módulos fotovoltaicos entre $8-15 \%$. Aplicaciones de fachadas situadas en los trópicos puede incluso experimentar una caída del $42 \%$ en el rendimiento, debido a ángulos de incidencia planas. Además, cuando es elevada temperatura de la célula de un módulo hay una disminución de $0.4 \%$ en el voltaje y la potencia de las celdas solares de silicio mono y policristalino: en referencia a la STC, ese número puede llegar al $20 \%[10]$.

Son innumerables los trabajos que se realizan en la modelación y diseño de paneles fotovoltaicos. En [11] un nuevo modelo térmico ha sido presentado para predecir el tiempo de respuesta de la temperatura de un panel fotovoltaico. El modelo ha sido validado por las mediciones de un panel fotovoltaico en condiciones de viento variable velocidades. El modelo incorpora las condiciones atmosféricas, las la composición del material del panel PV y la estructura de montaje.

Varios autores han modelado la temperatura media de salida a través de la radiación y convección, en una condición de estado estable, en el caso de la producción y la potencia generada [12].

El efecto pronunciado que la temperatura de funcionamiento de un módulo fotovoltaico (PV) tiene sobre su eficiencia eléctrica está bien documentado. Hay muchas correlaciones expresando Tc, la temperatura de la célula PV, como una función de variables meteorológicas tales como la temperatura ambiente, Ta, y la velocidad local del viento, Vw, así como la 
radiación solar flujo irradiancia, GT, con el material etc. Un número igualmente grande de las correlaciones que expresan la dependencia de la eficiencia eléctrica de la temperatura del módulo fotovoltaico [13].

Los datos de radiación solar proporcionan información sobre la cantidad de energía del sol golpea una superficie en un punto en la tierra durante un período de tiempo determinado. Estos datos son necesarios para la investigación eficaz en energía solar utilización. Debido al coste y la dificultad de en las mediciones de la radiación solar y estos datos no son fácilmente se necesitan disponibles formas, alternativas de la generación de estos datos [14].

\section{METODOLOGIA}

Se realiza el diseño, análisis, descripción y cálculo desde un punto de vista técnico-económico, de una instalación fotovoltaica conectada a la red de alimentación eléctrica para un frigorífico, cuyas características se describirán a continuación.

Los datos de partida necesarios para el dimensionado y cálculo de la instalación están constituidos por las condiciones de uso y las condiciones climáticas.

Las condiciones de uso son función de la demanda energética asociada a la instalación, según los diferentes tipos de consumo.

En cuanto a las condiciones climáticas, son función de la radiación global total en el campo de captación, la temperatura ambiente diaria y la temperatura del agua de la red.

Para los cálculos y diseño de la instalación se utilizó el programa PVSystem V6.42. Para [15], que es una herramienta para desarrollar instalaciones fotovoltaicas que permite el estudio, la simulación y análisis de datos completos de los sistemas fotovoltaicos.

Este software permite dimensionar el tamaño de las instalaciones teniendo en cuenta la radiación solar que recibiría en función de su ubicación gracias a su base de datos meteorológica, que permite su diseño en $3 \mathrm{D}$ y que tiene en cuenta la proyección de sombras gracias a la simulación del movimiento del sol durante el día.

También permite el análisis económico usando costes reales de componentes, costes adicionales y las condiciones de inversión, en cualquier moneda.

El software PVsyst V6.42 fue creado por el Grupo de Energía del Instituto de Ciencias Ambientales de la Universidad de Geneva, Switzerland por el Dr. André Mermoud, con el objetivo de facilitar el diseño e instalación de parques fotovoltaicos.

Los factores determinantes para el diseño, vienen en función de las coordenadas y altitud, son: orientación, inclinación, y estudio de sombras.

De los factores presentados, se tiene limitado uno de ellos, la orientación. Puesto que la disposición para la colocación de la planta generadora viene determinada por la ubicación impuesta por la edificación. Con ayuda del PVSystem V6.42, se selecciona la mejor inclinación y se puede realizar el estudio de las sombras.

El generador fotovoltaico está formado por un sub-campo, el mismo está compuesto por módulos fotovoltaicos, que a su vez, están formados por células fotovoltaicas. Las células en los módulos están conectadas en serie para que en su conjunto se pueda conseguir una mayor tensión en terminales del panel solar y entre ellas también en paralelo, para obtener una corriente adecuada para la conexión al inversor.

Con el programa PVSystem V6.42, se pueden conocer los datos de radiación anuales y los demás parámetros, expresados mensualmente de la ubicación de la instalación industrial.

La siguiente figura muestra la ventana principal del software PVsyst V6.42, el cual fue usado para el diseño de un parque solar fotovoltaico que pueda suplir las necesidades energéticas del frigorífico.

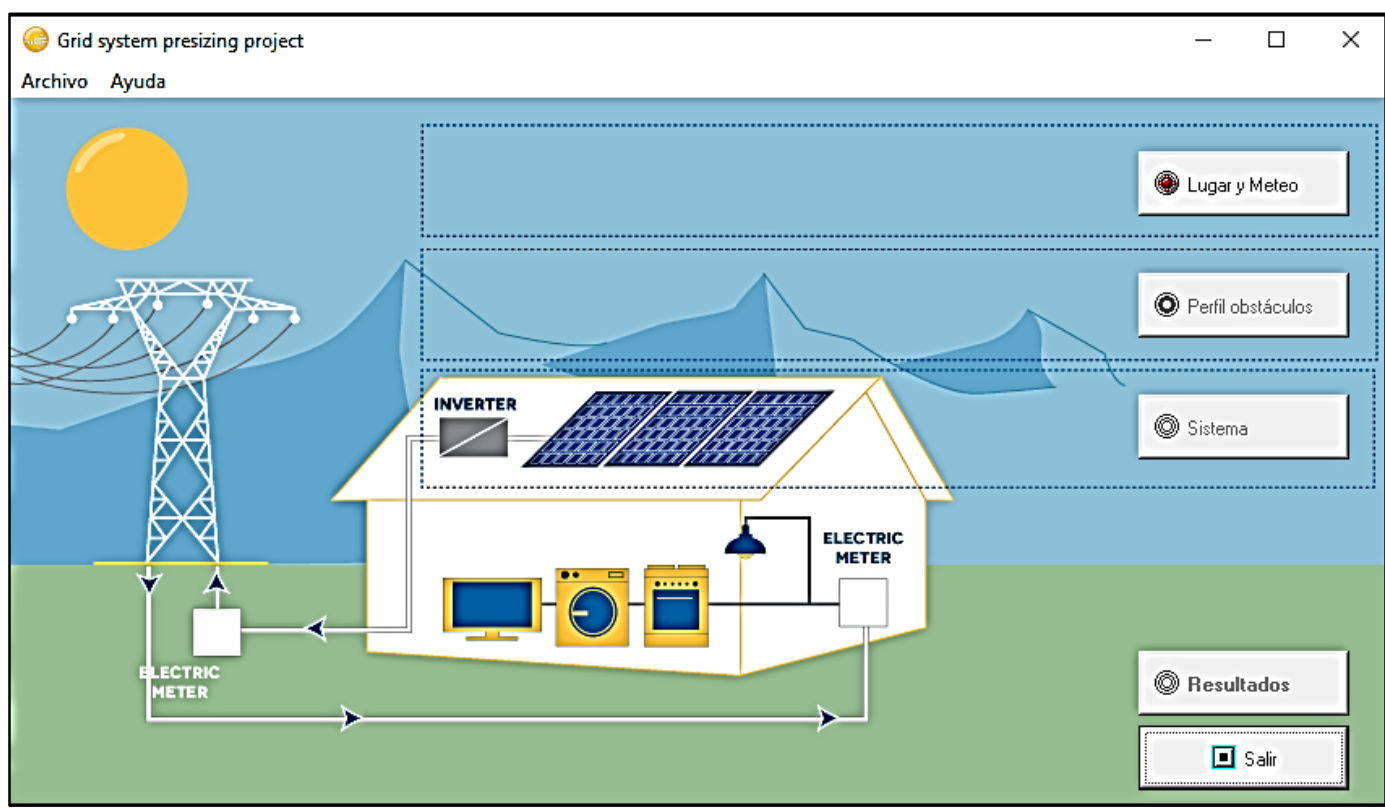

Figura 2: Ventana principal del software PVsyst V6.42.

Fuente: (Mermoud 2012). 


\section{ESTUDIO DE CASO}

La Empresa cuenta con una extensa nave donde se encuentran situadas 16 cámaras frigoríficas, que almacenan productos alimenticios, dicha nave posee un área techada de $14000 \mathrm{~m}^{2}$, además de $1600 \mathrm{~m} 2$ de área presentes en los techos de las oficinas, sería un total de $15600 \mathrm{~m} 2$ de área disponible para la instalación de paneles fotovoltaicos.
Se realiza un diagnóstico energético tomando los datos reportados por la entidad en el año 2015 y en el primer semestre del 2016, Figura 3 y 4.

El consumo mensual de energía eléctrica del Frigorífico se muestra a continuación en las próximas tablas.

\begin{tabular}{|c|c|c|c|c|c|c|c|c|c|c|c|c|}
\hline Año 2015 & \multicolumn{12}{|c|}{ Establecimiento 701} \\
\hline Mes & L.Mad. & L.Dia & L.Pico & C.Mad. & C.Dia & C.Pico & C.Perd. & C. Total & F.P. & $\mathrm{K}$ & Importe & Precio \\
\hline Enero & 557822 & 580957 & 22776 & 52053 & 47963 & 1755 & 2146,0 & 103917,0 & 0,86 & 5,1695 & $21.121,42$ & 203,25 \\
\hline Febrero & 602210 & 619951 & 24440 & 44388 & 38994 & 1664 & 1894,0 & 86940,0 & 0,86 & 5,5999 & $18.993,16$ & 218,46 \\
\hline Marzo & 659899 & 668132 & 26339 & 57689 & 48181 & 1899 & 2175,0 & 109944,0 & 0,86 & 4,7027 & $21.001,43$ & 191,02 \\
\hline Abril & 712629 & 733184 & 28101 & 52730 & 65052 & 1762 & 2162,0 & 121706,0 & 0,87 & 4,6031 & $23.011,89$ & 189,08 \\
\hline Mayo & 769960 & 796669 & 29995 & 57331 & 63485 & 1894 & 2241,0 & 124951,0 & 0,86 & 4,7805 & 81,14 & 191,12 \\
\hline Junio & 826071 & 855561 & 31700 & 56111 & 58892 & 1705 & 2155,0 & 118863,0 & 0,85 & 4,8218 & 6,29 & 192,80 \\
\hline Julio & 889146 & 922373 & 33794 & 63075 & 66812 & 2094 & 2297,0 & 134278,0 & 0,86 & 5,0601 & 29,74 & 194,59 \\
\hline Agosto & 952387 & 995114 & 35503 & 63241 & 72741 & 1709 & 2339,0 & 140030,0 & 0,85 & 5,0116 & $26.305,15$ & 187,85 \\
\hline Septiembre & 1011330 & 1082531 & 37206 & 58943 & 87417 & 1703 & 2341,0 & 150404,0 & 0,85 & 4,8773 & $27.971,06$ & 185,97 \\
\hline Octubre & 1073327 & 1162266 & 40100 & 61997 & 79735 & 2894 & 2403,0 & 147029,0 & 0,83 & 4,0424 & $24.780,88$ & 168,54 \\
\hline Noviembre & 1125986 & 1244039 & 41781 & 52659 & 81773 & 1681 & 2286,0 & 138399,0 & 0,83 & 4,1593 & $23.919,53$ & 172,83 \\
\hline Diciembre & 1180785 & 1326223 & 43398 & 54799 & 82184 & 1617 & 2292,0 & 140892,0 & 0,90 & 4,0844 & $24.026,61$ & 170,53 \\
\hline \multicolumn{4}{|c|}{ Total } & 675.016 & 793.229 & 22.377 & 26.731 & 1.517 .353 & 0,86 & & $284.058,28$ & 187,21 \\
\hline \multicolumn{4}{|c|}{ Promedio Mensual } & 56.251 & 66.102 & 1.865 & 2.228 & 126.446 & & & 23.671,52 & 188,8383 \\
\hline
\end{tabular}

Figura 3. Datos generales del consumo eléctrico del Frigorífico en el año 2015.

Fuente: Energético, (2015).

\begin{tabular}{|c|c|c|c|c|c|c|c|c|c|c|c|c|}
\hline Año 2016 & \multicolumn{12}{|c|}{ Establecimiento 701} \\
\hline Febrero & 1279688 & 1440134 & 46161 & 43965 & 52806 & 1281 & 1914,0 & 99966,0 & 0,93 & 4,1322 & $17.800,81$ & 178,07 \\
\hline Abril & 1383952 & 1581928 & 49585 & 55125 & 79362 & 1614 & 2201,0 & 138302,0 & 0,93 & 2,4219 & $18.733,71$ & 135,46 \\
\hline Mayo & 1436290 & 1668901 & 51102 & 52338 & 86973 & 1517 & 2291,0 & 143119,0 & 0,92 & 2,4332 & $19.406,80$ & 135,60 \\
\hline Junio & 1492234 & 1756878 & 53747 & 55944 & 87977 & 2645 & 2259,0 & 148825,0 & 0,92 & 0,0000 & $12.324,80$ & 82,81 \\
\hline \multicolumn{13}{|l|}{ Julio } \\
\hline \multicolumn{13}{|l|}{ Octubre } \\
\hline \multicolumn{13}{|l|}{ Noviembre } \\
\hline Diciembre & & & & & & & & & & & & \\
\hline \multicolumn{4}{|c|}{ Total } & 311.449 & 430.655 & 10.349 & 13.003 & 765.456 & 0,93 & & $109.715,75$ & 143,33 \\
\hline & Promedio I & Mensual & & 25.954 & 35.888 & 862 & 2.167 & 127.576 & & & $18.285,96$ & 147,3936 \\
\hline
\end{tabular}

Figura 4. Datos generales del consumo eléctrico del Frigorífico hasta la fecha en el año 2016.

Fuente: Energético, (2016).

De acuerdo al análisis de las tablas anteriores se tiene que el consumo anual de esta instalación es aproximadamente de 1600 MW-h/año, previendo un consumo superior en los próximos años, se propone diseñar una planta de generación fotovoltaica con un rendimiento anual de $2000 \mathrm{MW}-\mathrm{h} / \mathrm{año}$.

\section{III.1. CÁLCULOS Y DIMENSIONADO}

Seguidamente al pulsar el botón (Resultados) de la ventana principal del software, una de las opciones que aparecen es la de la tabla a continuación, que muestra cómo se comportaría mensualmente la irradiación solar y la generación de energía eléctrica por los paneles fotovoltaicos seleccionados. 


\begin{tabular}{|c|c|c|c|c|}
\hline & $\begin{array}{l}\text { Gl. horiz. } \\
\mathrm{kWh} / \mathrm{m}^{2} \text {.dia }\end{array}$ & $\begin{array}{l}\text { Coll. Plane } \\
\mathrm{k} / \mathrm{h} / \mathrm{m}^{\mathbf{2}} \text {. dia }\end{array}$ & $\begin{array}{c}\text { System output } \\
\text { kWh/dia }\end{array}$ & $\begin{array}{c}\text { System output } \\
\mathrm{K} W / \mathrm{h}\end{array}$ \\
\hline Ene. & 3.82 & 5.21 & 5375 & 166626 \\
\hline Feb. & 4.44 & 5.41 & 5588 & 156472 \\
\hline Mar. & 5.45 & 6.00 & 6194 & 192008 \\
\hline Abr. & 6.20 & 6.04 & 6239 & 187155 \\
\hline May. & 6.22 & 5.49 & 5671 & 175812 \\
\hline Jun. & 5.76 & 4.89 & 5044 & 151332 \\
\hline Jul. & 6.16 & 5.27 & 5445 & 168793 \\
\hline Ago. & 5.88 & 5.46 & 5638 & 174779 \\
\hline sep. & 5.04 & 5.25 & 5417 & 162520 \\
\hline Oct. & 4.25 & 4.95 & 5110 & 158416 \\
\hline Nov. & 3.87 & 5.04 & 5209 & 156276 \\
\hline Dic. & 3.39 & 4.68 & 4833 & 149606 \\
\hline An̂o & 5.04 & 5.31 & 5479 & 2000000 \\
\hline
\end{tabular}

Figura 5. Comportamiento mensual de la irradiación solar y la generación de energía eléctrica por los paneles fotovoltaicos.

Fuente: Los Autores, (2016).

Los datos de la tabla anterior fueron tomados para confeccionar gráficos de barra los cuales suelen ser más ilustrativos.

El gráfico de la figura 6 a continuación describe el comportamiento mensual de la irradiación solar en $\mathrm{kW}-\mathrm{h} / \mathrm{m} 2$ día en una línea horizontal global (rojo) y en un plano inclinado global (verde).

Al finalizar el año la media de la línea horizontal global es de $5 \mathrm{~kW}-\mathrm{h} / \mathrm{m} 2$ día y la del plano inclinado global es de $5.3 \mathrm{~kW}$ $\mathrm{h} / \mathrm{m} 2$ día.

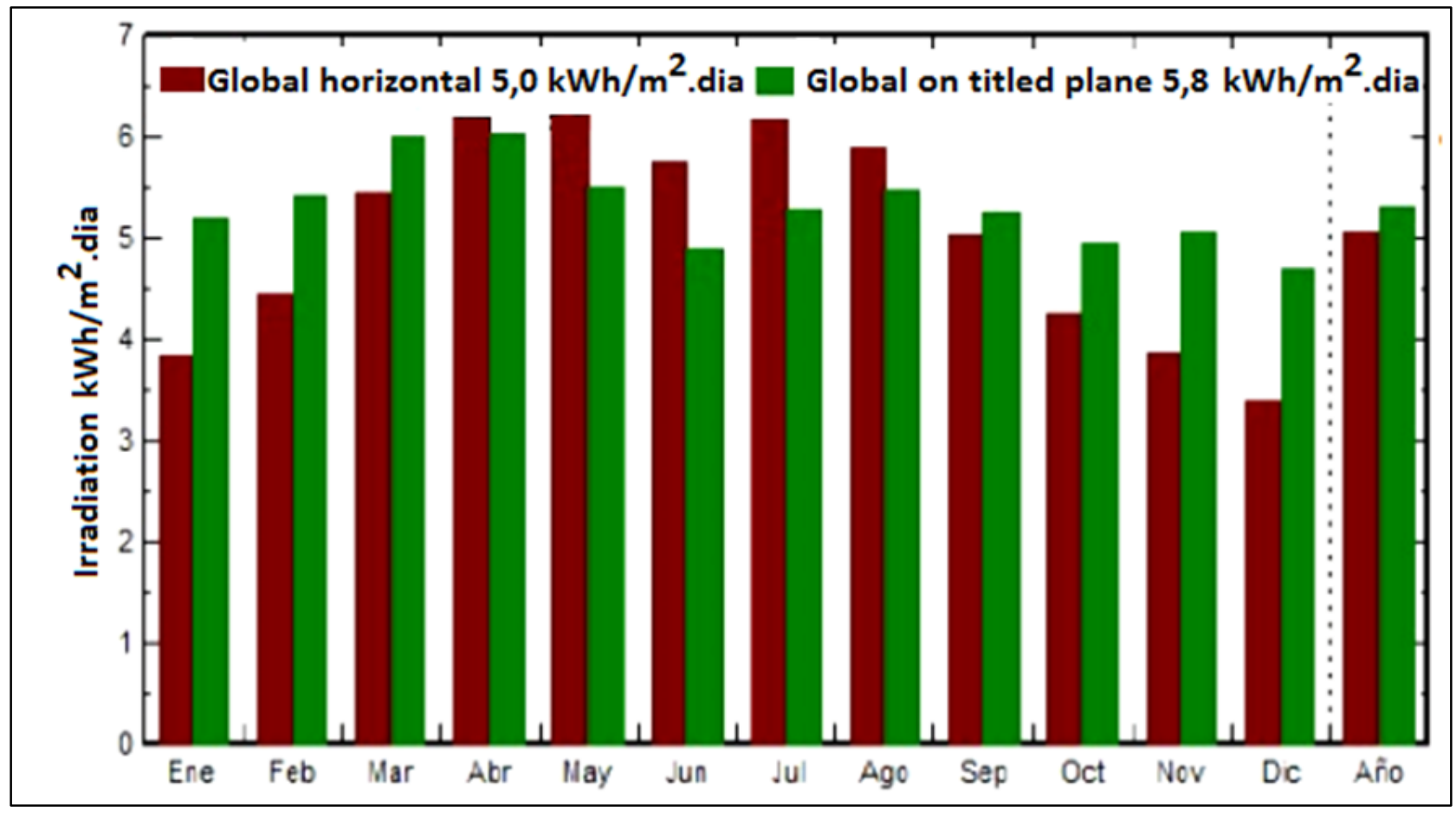

Figura 6: Gráfico del Comportamiento mensual de la irradiación solar en $\mathrm{kW}-\mathrm{h} / \mathrm{m}^{2}$ día. Fuente: Los Autores a partir del PVSystem, (2016). 
El siguiente grafico de la figura 7 describe el comportamiento mensual de la generación de energía eléctrica en
$\mathrm{kW}-\mathrm{h} / \mathrm{m}^{2}$ día. Al finalizar el año se obtiene el resultado esperado de $2000 \mathrm{MW}-\mathrm{h} / \mathrm{año.}$

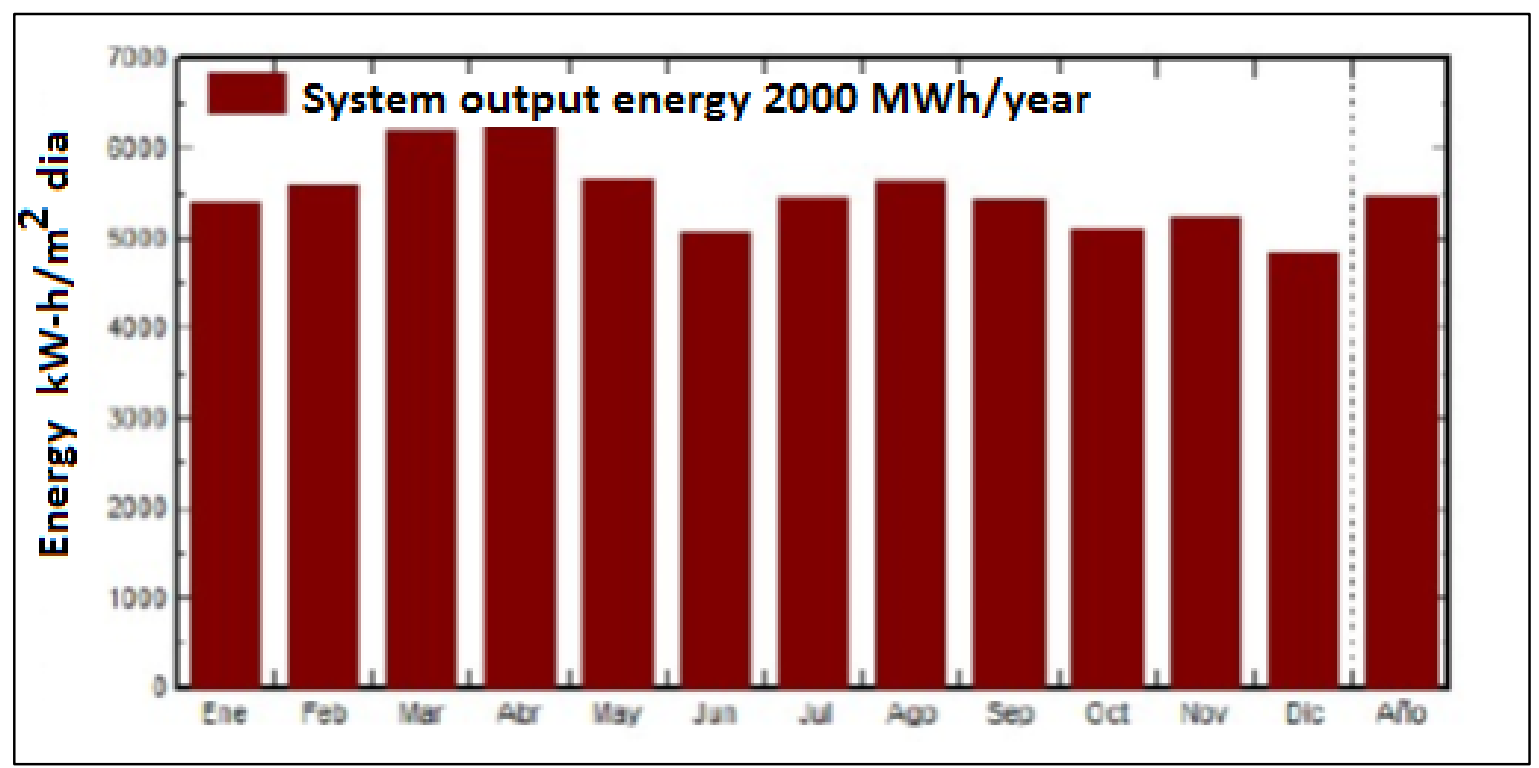

Figura 7: Gráfico del Comportamiento mensual de la generación de energía en kW-h/m² día. Fuente: Los Autores a partir del PVSystem, (2016).

\section{III.2 CARACTERÍSTICAS DEL MÓDULO FOTOVOLTAICO}

El conjunto de paneles está conectado a 2 inversores de $500 \mathrm{~kW}$, según se muestra en la figura 8.

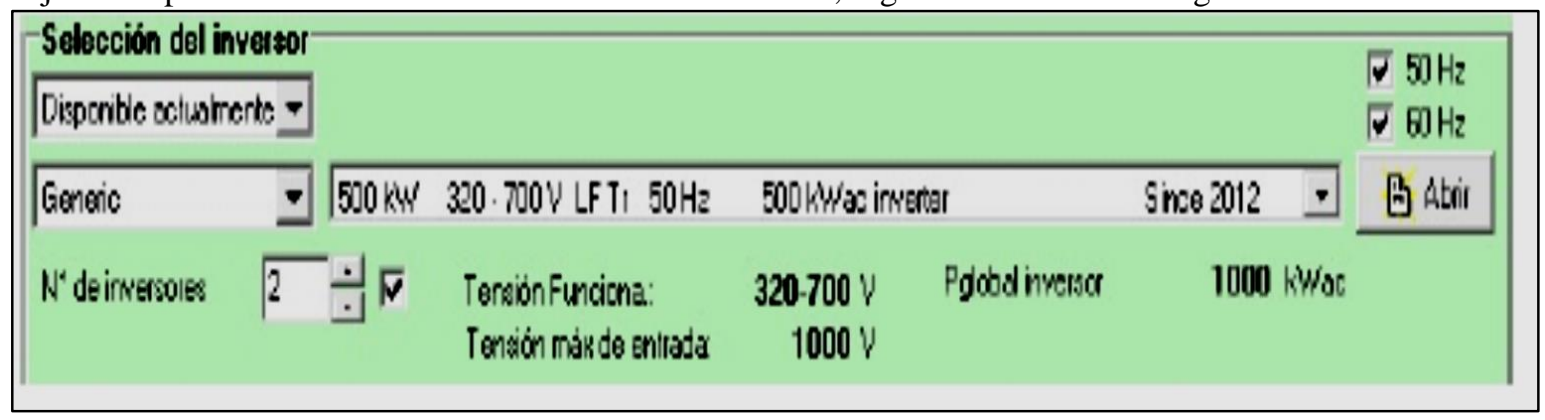

Figura 8. Características del inversor para el parque fotovoltaico

Fuente: Autores a partir del PVSystem, (2016).

En las figuras 9 y 10 se muestran las dimensiones del panel fotovoltaico seleccionado así como las especificaciones dadas por el fabricante para dicho panel fotovoltaico.

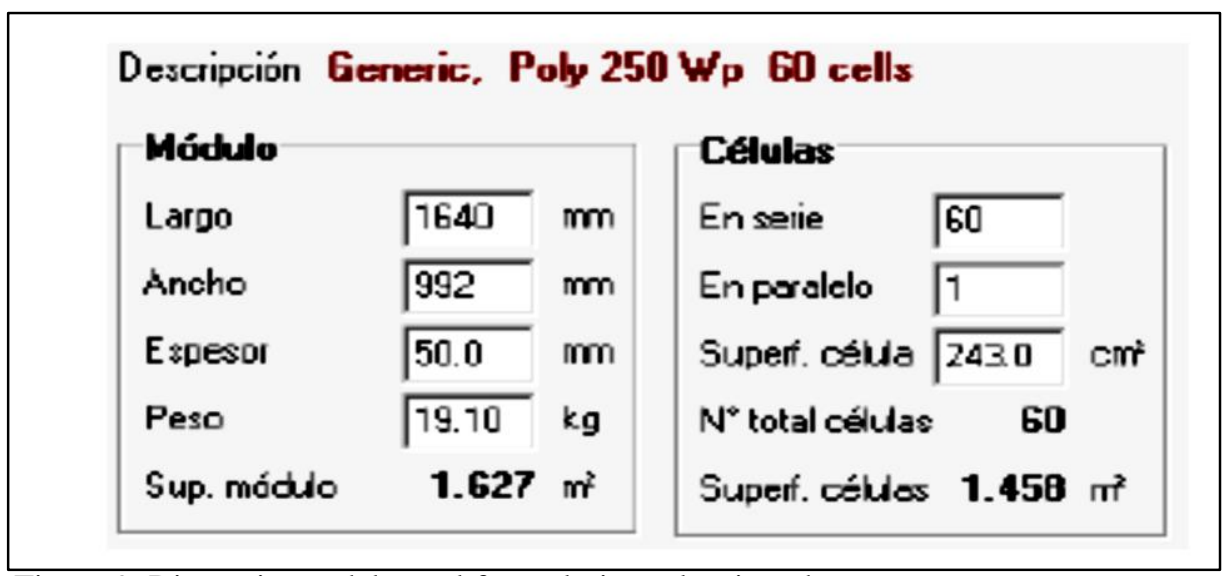

Figura 9. Dimensiones del panel fotovoltaico seleccionado.

Fuente: Autores a partir del PVSystem, (2016). 


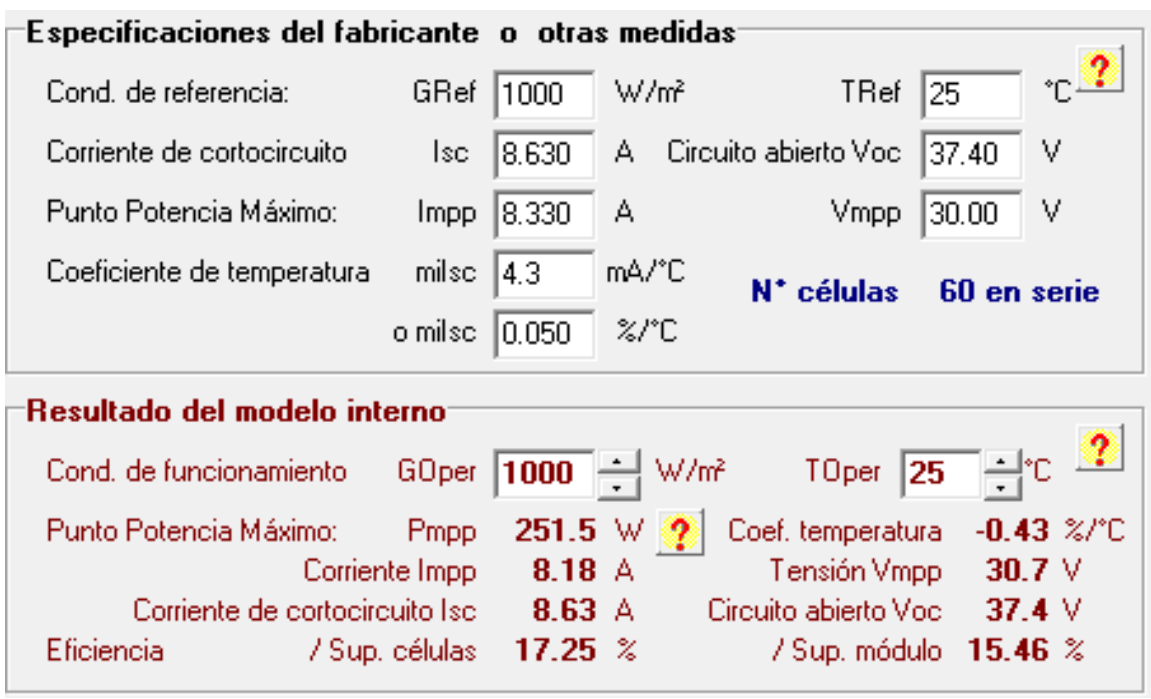

Figura 8. Especificaciones del fabricante del panel fotovoltaico seleccionado. Fuente: Autores a partir del PVSystem, (2016).

\section{ANÁLISIS DE LOS RESULTADOS \\ IV.1. EVALUACIÓN ECONÓMICA}

El análisis económico de un proyecto es fundamental, no solo para determinar la conveniencia de efectuar una inversión, sino también para predecir el posible comportamiento de la misma, pudiendo así evitar o limitar perjuicios económicos importantes para los inversionistas. Además, que las entidades financieras exigen este tipo de estudios antes de aprobar los créditos que se requieran para la ejecución de cualquier tipo de actividad.
Mediante el software PVsyst V6.42 se calcula, Figura 9, que el parque solar fotovoltaico para el

Frigorífico de Santa Clara tendría una inversión total de \$ 4132076 US o de 3029381 EUR. La ventana muestra además el costo de cada módulo fotovoltaico $(0.95$ US \$/ Wp) y realiza una evaluación económica general de las principales inversiones. Se observa que la inversión tendría un periodo de recuperación de 8 años asociado al costo de venta de la energía eléctrica de $(0.25$ EUR/ kW-h).

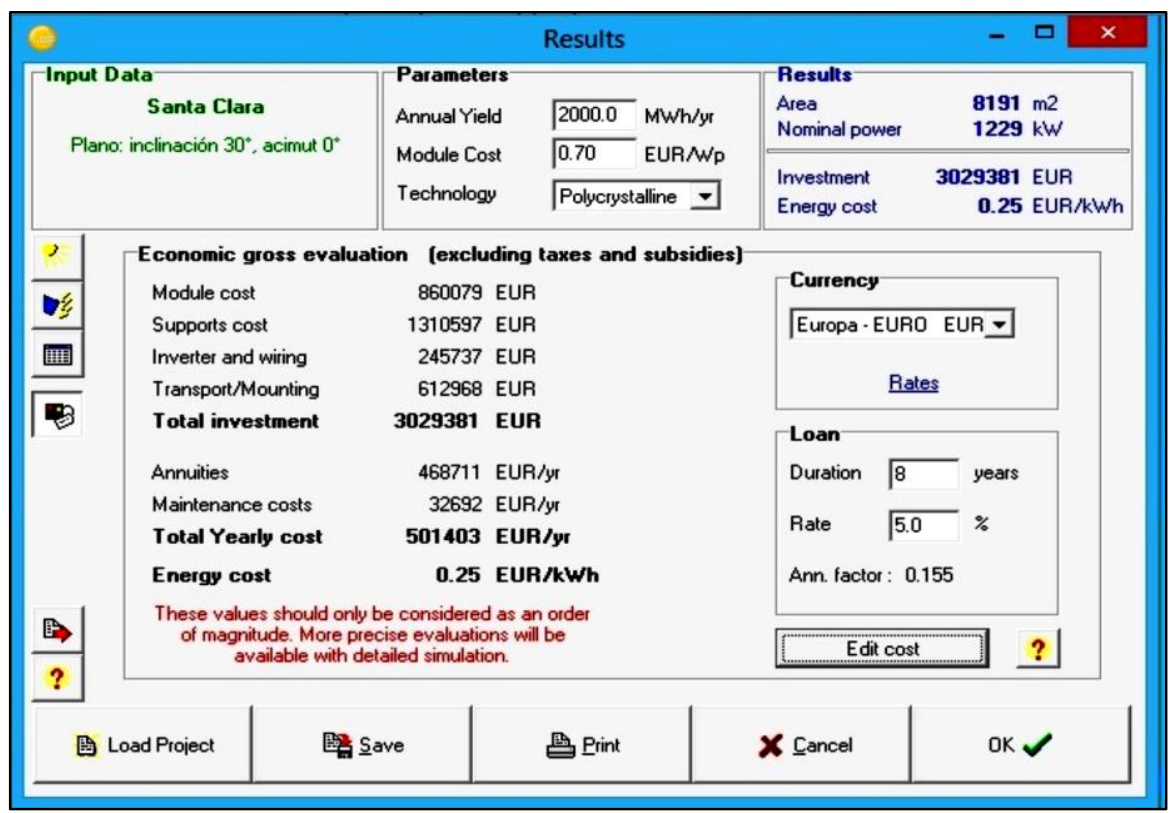

Figura 9. Estimado de los costos de inversión general de los paneles diseñados, en Euros. Fuente: Autores a partir del PV System, (2016).

\section{IV.2 ANÁLISIS DEL AHORRO EN USD DE PETRÓLEO NO CONSUMIDO:}

Con los siguientes precios e índices:

Precio Fuel oíl: 743.USD/Ton

Precio Electricidad: 0.267USD/kWh

Consumo específico neto de combustible: $256.5 \mathrm{~g} / \mathrm{kWh}$
El proyecto ahorrará 552 toneladas por año de petróleo lo que representa un ahorro de 410 279,5 USD al año.

En el siguiente análisis, se mostrará una previsión del flujo de efectivo de la instalación durante los 25 años de vida útil estimada, se simula el flujo de caja anual y se estimarán los 
parámetros VAN (Valor Actual Neto) y TIR (Tasa Interna de Retorno), estos son indicadores utilizados para saber la viabilidadb de la inversión.

El VAN nos proporciona una medida absoluta de rentabilidad de la instalación. Un VAN obtenido

positivo nos indica que la instalación crea valor, pudiendo ser abordada. En caso contrario, VAN

negativo, la instalación generará perdidas y no es interesante la inversión.

$$
V A N=I T+\sum_{i=1}^{n} \frac{F_{i}}{(1+r)_{i}}
$$

donde: IT = Inversión total, Fi = Flujos de caja en cada año del proyecto. (Ingresos - costos),

$\mathrm{n}=$ vida útil, $\mathrm{r}=$ tasa de actualización.

A continuación, se muestran los criterios para evaluar la inversión en función del VAN:

Si VAN>0 La Instalación es rentable

$\mathrm{Si} \mathrm{VAN}=0 \mathrm{El}$ proyecto no agrega valor monetario por encima de la rentabilidad exigida

Si VAN $<0$ La instalación no es rentable

TIR: Tasa interna de rentabilidad.

La tasa interna de retorno o tasa interna de rentabilidad (TIR) de una inversión es la media geométrica de los rendimientos futuros esperados de dicha inversión, y que implica por cierto el supuesto de una oportunidad para "reinvertir" [15].

A continuación se brindan los resultados de los indicadores económicos que se calcularon el VAN y el TIR

para lo que se fue necesario calcular el costo de operación, los ingresos y otros indicadores todo lo cual fue programado en una hoja de cálculo en Excel.

Los ingresos se asumen por daños evitados (externalidades), para cuantificarlos se tuvieron en cuenta los siguientes aspectos:

- Daños evitados a las personas y al medioambiente por no emisión de $\mathrm{CO}_{2}$, ahorro por no pago de multas ambientales.

Petróleo dejado de consumir.

Venta de electricidad a la Red Nacional.

Según los valores obtenidos para el VAN $\$ 1,880.926$ y una TIR 18\%, la inversión propuesta es viable desde el punto de vista económico, se recupera aproximadamente en 9 años, además, se pueden aceptar variaciones de hasta un $10 \%$ de disminución de los ingresos, y hasta un $10 \%$ de aumento de la inversión.

\section{IV.3. IMPACTO AMBIENTAL}

La influencia humana ha sido detectada en el calentamiento de la atmósfera y el océano, en los cambios en el ciclo mundial del agua, en las reducciones en la nieve y el hielo, en el aumento del nivel medio global del mar, y en los cambios extremas en el clima. Es muy probable que la influencia humana ha sido la causa principal del calentamiento observado desde la mitad del siglo 20. Esta influencia humana se hace relevante en el aumento de las concentraciones de $\mathrm{CO}_{2}$ en la atmósfera terrestre [16].

De igual forma el software PVsyst V6.42 brinda información de las emisiones de $\mathrm{CO}_{2}$ que se dejan de producir por ahorro de combustibles fósiles, al no utilizar petróleo como combustible reportándose los valores en t de combustible no consumido para la vida útil considerada de la instalación. El proyecto del Parque solar fotovoltaico logrará la reducción de emisiones de $\mathrm{CO}_{2}$, pues se disminuye el consumo de petróleo. El $\mathrm{CO}_{2}$ es el Gas de Efecto Invernadero (GEI) más importante, y el más común producido por las actividades humanas. Es el que más contribuye al calentamiento global, aporta cerca de $63 \%$ del incremento en el forzamiento radiativo total de los Gases Mayores de Efecto Invernadero de Larga Vida GEILV en la época industrial.

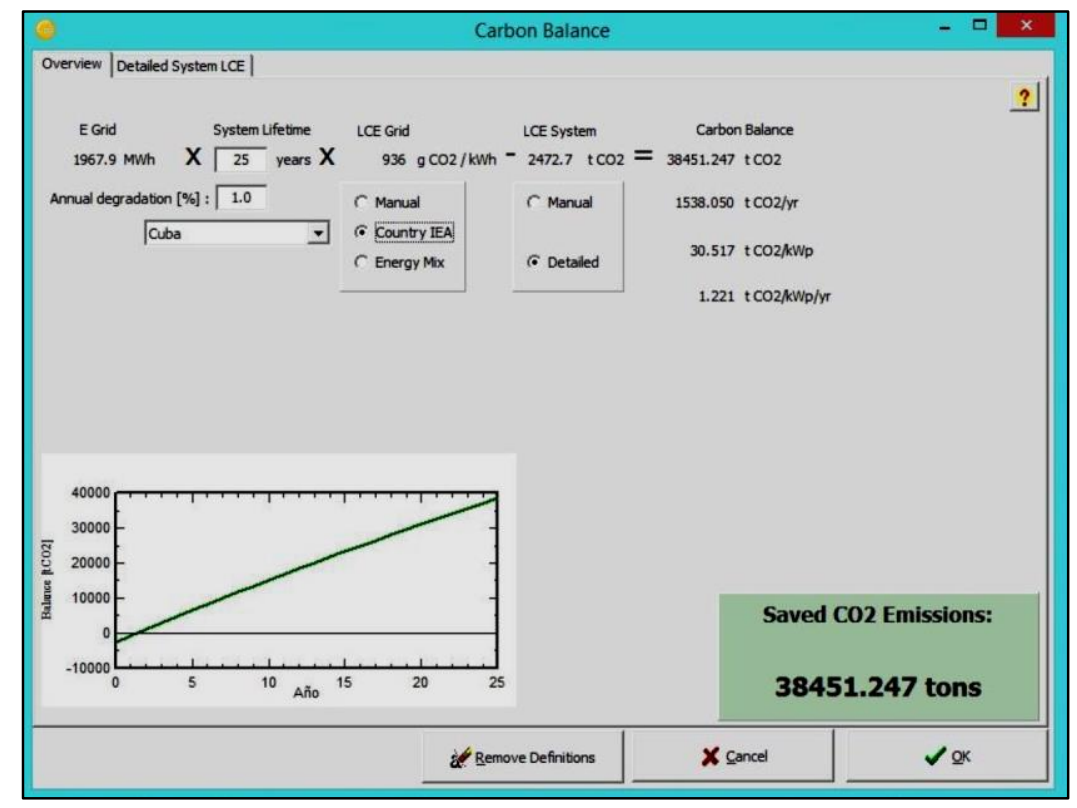

Figura 10. Balance de $\mathrm{CO}_{2}$ del proyecto propuesto.

Fuente: Autores a partir del PV System, (2016). 


\section{CONCLUSIONES}

Se diseñó un sistema de paneles fotovoltaicos que responden a la capacidad requerida por la Unidad tomada como estudio de caso, de 2000MW-h/año, con las siguientes características 5040 módulos fotovoltaicos, cada panel compuesto por 60 células fotovoltaicas de silicio policristalino con una potencia máxima de $250 \mathrm{Wp}$ y dos inversores de $500 \mathrm{~kW}$ cada uno.

Se logra una tecnología amigable con el medioambiente pues se evita la emisión a la atmosfera de 38 451,0t de CO2 en un período de 25años y se dejan de utilizar 13800 t de petróleo. La propuesta de inversión tiene un valor de \$4 132076 US.

La evaluación económica demuestra la factibilidad de la propuesta, con un valor del VAN $\$ 1,880.926$ y una TIR $18 \%$, y un período de recuperación de aproximadamente 9 años.

\section{REFERENCIAS BIBLIOGRÁFICAS}

[1] Isabella, O., et al. (2016). Solar Energy: The physics and engineering of photovoltaic conversion, technologies and systems, UIT Cambridge Limited

[2] Mehleri, E., et al. (2010). "Determination of the optimal tilt angle and orientation for solar photovoltaic arrays." Renewable Energy 35(11): 2468-2475.

[3] Martins, D. C. and R. Demonti (2000). Interconnection of a photovoltaic panels array to a single-phase utility line from a static conversion system. Power Electronics Specialists Conference, 2000. PESC 00. 2000 IEEE 31st Annual, IEEE.

[4] Notton, G., et al. (2005). "Modelling of a double-glass photovoltaic module using finite differences." Applied Thermal Engineering 25(17): 2854-2877.

[5] Hussein, H., et al. (2004). "Performance evaluation of photovoltaic modules at different tilt angles and orientations." Energy conversion and management 45(15): 2441-2452.

[6] Bialasiewicz, J. T. (2008). "Renewable energy systems with photovoltaic power generators: Operation and modeling." IEEE Transactions on industrial Electronics 55(7): 2752-2758.

[7] Salas, V., et al. (2006). "Review of the maximum power point tracking algorithms for stand-alone photovoltaic systems." Solar energy materials and solar cells 90(11): 15551578.

[8] Kacira, M., et al. (2004). "Determining optimum tilt angles and orientations of photovoltaic panels in Sanliurfa, Turkey." Renewable Energy 29(8): 1265-1275.

[9] Moshfegh, B. and M. Sandberg (1998). "Flow and heat transfer in the air gap behind photovoltaic panels." Renewable and Sustainable Energy Reviews 2(3): 287-301.
[10] Krauter, S. (2004). "Increased electrical yield via water flow over the front of photovoltaic panels." Solar energy materials and solar cells 82(1): 131-137.

[11] Armstrong, S. and W. Hurley (2010). "A thermal model for photovoltaic panels under varying atmospheric conditions." Applied Thermal Engineering 30(11): 1488-1495.

[12] Jones, A. and C. Underwood (2001). "A thermal model for photovoltaic systems." Solar energy 70(4): 349-359.

[13] Skoplaki, E. and J. Palyvos (2009). "On the temperature dependence of photovoltaic module electrical performance: A review of efficiency/power correlations." Solar energy 83(5): 614-624.

[14] Khatib, T., et al. (2012). "A review of solar energy modeling techniques." Renewable and Sustainable Energy Reviews 16(5): 2864-2869.

[15] Peters, M. S.; Timmerhaus, K. D.; West, R. E.; Timmerhaus, K.; West, R. Plant design and economics for chemical engineers. McGraw-Hill New York, 1968.

[16] Mermoud, A. (2012). "Pvsyst: Software for the study and simulation of photovoltaic systems." ISE, University of Geneva, www. pvsyst. com.

[17] Commission, I. E. (2008). "IEC 61730-2 Photovoltaic (PV) Module Safety Qualification-Part 2: Requirements for Testing." International Electrotechnical Commission: Geneva. 